\title{
Role of sodium bicarbonate in the management of acute upper gastrointestinal bleeding
}
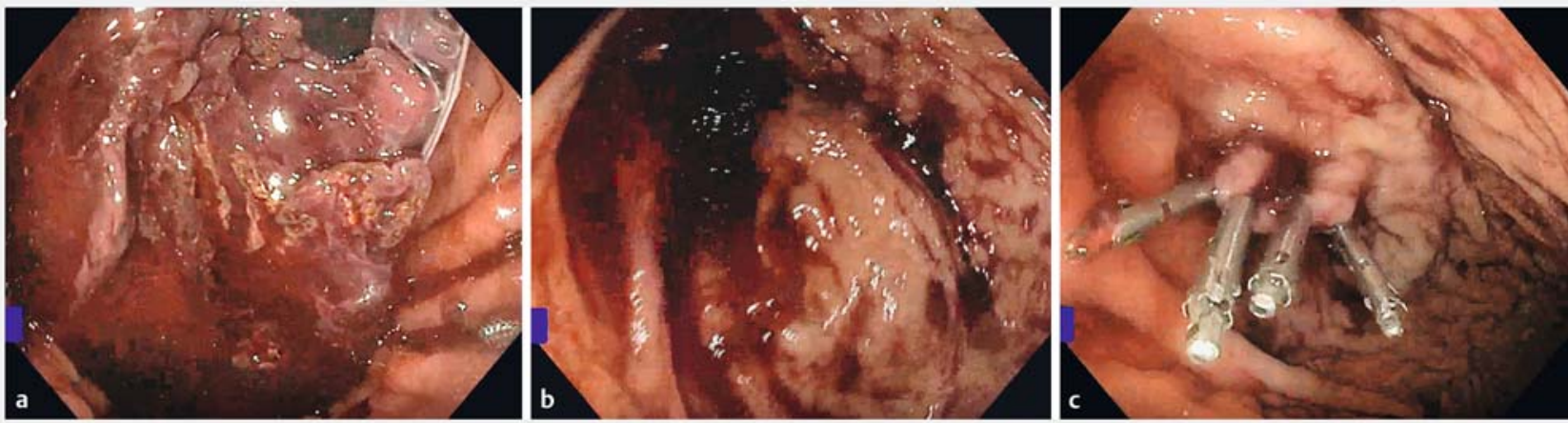

- Fig. 1 Endoscopic views in a 29-year-old man with coffee-ground emesis and hematochezia showing: a adherent clot at the gastric fundus visualized in retroflexion with a Salem sump tube visible; $\mathbf{b}$ the gastric fundus post-sodium bicarbonate irrigation; $\mathbf{c}$ the lesion after successful hemostatic therapy with epinephrine and hemostatic clips.

Sodium bicarbonate has previously been reported to have effects on blood clot formation both in vitro and in vivo. It is thought that sodium bicarbonate interferes with fibrin clot formation $[1,2]$. We describe our experience with two patients with upper gastrointestinal (GI) bleeding in whom sodium bicarbonate was used to improve visualization, thus allowing definitive control of the bleeding.

Case 1 was a 29-year-old man with a complex past medical history including single morphologic right ventricle, unbalanced right dominant atrioventricular septal defect, and D-transposed great arteries who was admitted to our facility. The patient was noted to have coffee-ground emesis and hematochezia. Because of concerns about upper Gl bleeding, the gastroenterology department was asked to perform endoscopic evaluation at the bedside. Visualization of the underlying mucosa was difficult owing to a large adherent clot ( $\triangleright$ Fig. 1 a; $\triangleright$ Video 1 , time 00:26). Subsequent irrigation with sterile water over the next 2 hours failed to improve visualization. A total of $100 \mathrm{~mL}$ of $8.4 \%$ sodium bicarbonate solution was applied intermittently with subsequent improvement in visualization ( $\mathbf{F i g . 1} \mathbf{b}$ ).

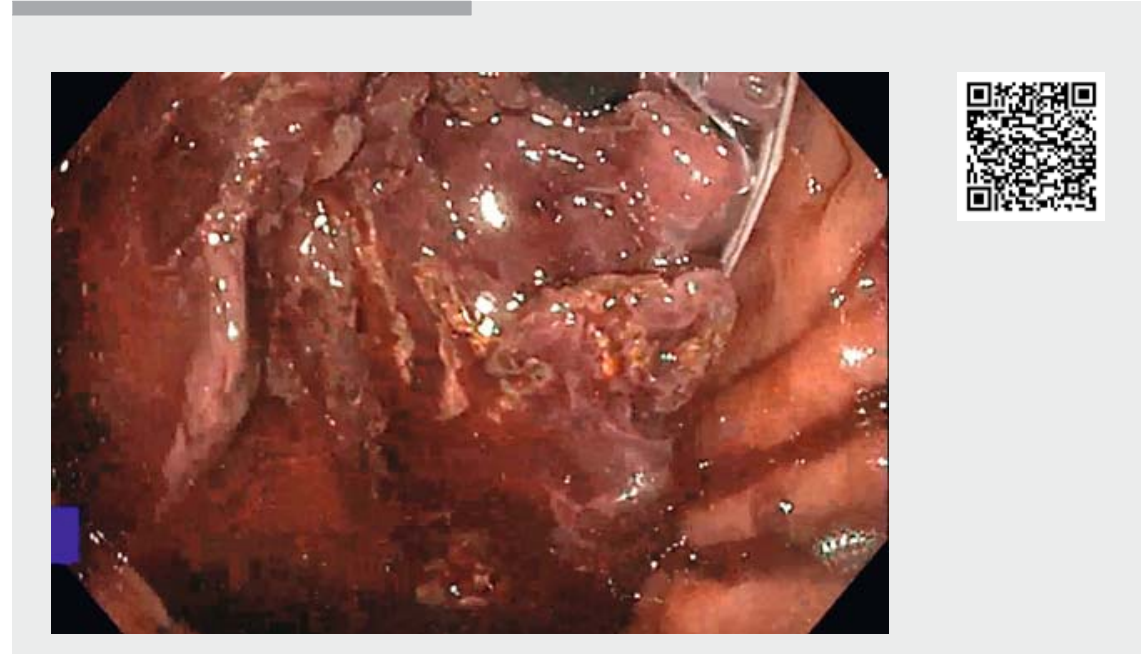

$\checkmark$ Video 1 Use of sodium bicarbonate solution in the management of two patients with acute upper gastrointestinal bleeding.

Following this process, a Dieulafoy lesion was identified and was managed with epinephrine and hemostatic clips ( $>$ Fig. $1 \mathrm{c})$.

Case 2 was a 3-year-old boy with a past medical history of disseminated medulloblastoma, for which he had undergone gross total resection and craniospinal radiation, who presented with hematemesis. Upper GI endoscopy was performed urgently. Visual findings were significant for a flat, pigmented ulcer at the level of the gastroesophageal junction ( $\vee$ Video 1, time 01:40). Hemostasis was not performed given lack of stigmata of recent bleeding. Approximately 48 hours after the initial procedure, the patient was noted to have a drop in hemoglobin from 10 to $5 \mathrm{~g} / \mathrm{dL}$. The patient was again taken to operating room for endoscopic 

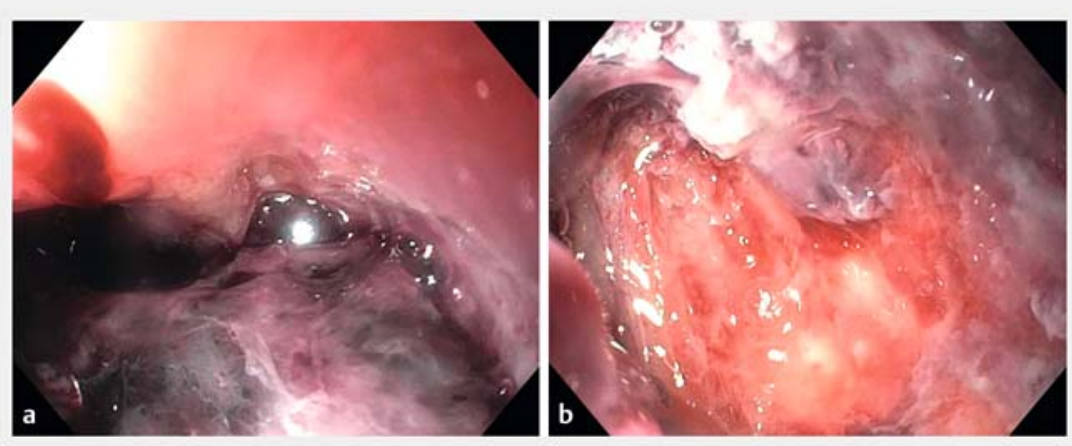

- Fig. 2 Endoscopic views in a 3-year-old boy who presented with hematemesis showing: $\mathbf{a}$ an area of known ulceration obstructed by a large adherent clot; $\mathbf{b}$ the ulcer bed after sodium bicarbonate irrigation.

evaluation. As the endoscope was passed into the stomach, a large clot was found to be obscuring the previously noted ulcer base ( $\triangleright$ Fig.2a). Epinephrine was injected at the periphery of the ulcer and sodium bicarbonate was then used topically to dissolve the clot. Following multiple rounds of irrigation with sodium bicarbonate and sterile water, a full view of the ulcer bed was obtained ( $\mathbf{F i g} \cdot \mathbf{2} \mathbf{b}$ ). There did not appear to be any additional bleeding. A total of $40 \mathrm{~mL}$ of $8.4 \%$ sodium bicarbonate solution $(1 \mathrm{meq} / \mathrm{mL})$ were used in aliquots of $4-10 \mathrm{~mL}$ without any immediate side effects.

Prior cases have documented the use of sodium bicarbonate for severe clot retention in the bladder; to our knowledge, this is the first report to describe its use in the management of a GI bleed [3]. This was particularly advantageous given poor visualization and concern for clot instability. While caution should be exercised owing to the anticoagulant properties of sodium bicarbonate, this technique can be a useful modality combined with traditional methods of hemostasis [4].

Endoscopy_UCTN_Code_TTT_1AO_2AD

\section{References}

[1] Wong DW. Effect of sodium bicarbonate on in vitro conversion of fibrinogen to fibrin. J Pharm Sci 1980; 69: 978-980

[2] Wong DW, Mishkin FS, Tanaka TT. The effects of bicarbonate on blood coagulation. JAMA 1980; 244: 61-62

[3] Bo J, Yangyang Y, Jiayuan L et al. Evaluation of bladder clots using a nonsurgical treatment. Urology 2014; 83: 498-499

[4] Lightdale JR, Acosta R, Shergill AK et al. Modifications in endoscopic practice for pediatric patients. Gastrointest Endosc 2014; 79: 699-710

Bibliography

Endoscopy 2021; 53: E155-E156

DOI 10.1055/a-1220-5957

ISSN 0013-726X

published online 20.8.2020

(c) 2020. Thieme. All rights reserved.

The authors declare that they have no conflict of interest.

Georg Thieme Verlag KG, Rüdigerstraße 14, 70469 Stuttgart, Germany

The authors

Jonathan Wong1 ${ }^{1}$, Susan Taylor ${ }^{2}$, Diana Lerner ${ }^{1}$

1 Division of Pediatric Gastroenterology, Hepatology and Nutrition, Department of Pediatrics, Medical College of Wisconsin, Milwaukee, Wisconsin, USA

2 Division of Pediatric Anesthesia, Department of Pediatrics, Medical College of Wisconsin, Milwaukee, Wisconsin, USA

\section{Corresponding author}

\section{Jonathan Wong, DO}

Division of Gastroenterology, Hepatology and Nutrition, Medical College of Wisconsin, 8701 Watertown Plank Road, Milwaukee, WI 53226, USA

Fax: +1-414-266-8495

jwong@mcw.edu

\section{ENDOSCOPY E-VIDEOS \\ https://eref.thieme.de/e-videos}

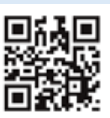

Endoscopy E-Videos is a free access online section, reporting on interesting cases and new

techniques in gastroenterological endoscopy. All papers include a high quality video and all contributions are freely accessible online.

This section has its own submission website at https://mc.manuscriptcentral.com/e-videos 